\title{
Absence of Singular Continuous Diffraction for Discrete Multi-Component Particle Models
}

\author{
Michael Baake • Natali Zint
}

Received: 6 August 2007 / Accepted: 19 September 2007 / Published online: 13 November 2007

(c) to the author 2007

\begin{abstract}
Particle models with finitely many types of particles are considered, both on $\mathbb{Z}^{d}$ and on discrete point sets of finite local complexity. Such sets include many standard examples of aperiodic order such as model sets or certain substitution systems. The particle gas is defined by an interaction potential and a corresponding Gibbs measure. Under some reasonable conditions on the underlying point set and the potential, we show that the corresponding diffraction measure almost surely exists and consists of a pure point part and an absolutely continuous part with continuous density. In particular, no singular continuous part is present.
\end{abstract}

Keywords Diffraction theory $\cdot$ Lattice gases · Model sets · Gibbs measures

\section{Introduction}

Periodic systems in general, and lattice systems in particular, are at the basis of crystallographic structure determination from (kinematic) diffraction data. The diffraction spectrum (or measure) of a crystallographic (or fully periodic) system in $\mathbb{R}^{d}$, with lattice of periods $\Gamma$, is a positive pure point measure, concentrated on the dual lattice $\Gamma^{*}$, the latter defined as $\Gamma^{*}=\left\{z \in \mathbb{R}^{d} \mid z \cdot y \in \mathbb{Z}, \forall y \in \Gamma\right\}$.

In reality, however, crystals are never perfect, and deviations from full periodicity are abundant. One interesting class of extensions to consider is that of the so-called lattice gases, where different types of atoms (or one type and "zeronium") occupy the lattice sites according to some random process, e.g., one described by a Gibbs measure. The relevance of Gibbs measures, in turn, stems from the assumption that the structure under consideration is in thermal equilibrium. Clearly, this need not always be the case, but we do not consider non-equilibrium systems here.

M. Baake $(\bowtie) \cdot$ N. Zint

Fakultät für Mathematik, Universität Bielefeld, Postfach 100131, 33501 Bielefeld, Germany

e-mail: mbaake@math.uni-bielefeld.de

N. Zint

e-mail: nzint@math.uni-bielefeld.de 
In two recent publications [6, 14], it was shown that certain binary lattice gases, such as those based upon a ferromagnetic Ising model with short-range interaction, additionally inherit an absolutely continuous spectral component, but no singular continuous one. This finding is in agreement with the working experience in crystallography (compare [9, 12, 26]), and the natural question arises whether this situation is more general.

The answer to this question is affirmative, and it is the aim of this contribution to extend the results of [6] to increase the evidence for the rather natural conjecture that non-trivial singular continuous diffraction spectra are the exception, at least as the result of stochastic deviations from systems with a strongly ordered ground state, which are of special interest (see [10]).

Starting with a brief summary of the concept of finite local complexity (FLC), we show that, under some condition on the potential (such as finite or short range) and sufficiently high temperature, there is no singular continuous diffraction component for a lattice gas with finitely many types of particles. Our aim here is a simultaneously more general and more transparent approach in comparison to [4, 6], where only binary systems were considered. Also, the focus is different both from [17], where an approach via finite subsystems is used for the interaction free case, and from [18], where a large deviation estimate for the diffraction of a finite point set is derived.

Finally, after various explicit examples in Sect. 4, we prove an analogous result for a certain class of FLC sets in Sect. 5.

\section{FLC Sets}

In what follows, we consider only point sets $\Gamma \subset \mathbb{R}^{d}$ that are locally finite, i.e., the intersection $\Gamma \cap K$ with any compact $K \subset \mathbb{R}^{d}$ is a finite set. Such a $\Gamma$ has finite local complexity (FLC), if, for any compact set $K \subset \mathbb{R}^{d}$, there are only finitely many clusters $\Gamma \cap(t+K)$, $t \in \mathbb{R}^{d}$, when counted up to translations. By a characterization of Schlottmann [24], a point set $\Gamma \subset \mathbb{R}^{d}$ is FLC if and only if $\Gamma-\Gamma:=\{x-y \mid x, y \in \Gamma\}$ is discrete and closed (or locally finite). This characterization automatically includes the local finiteness of $\Gamma$. Clearly, FLC sets $\Gamma$ are also uniformly discrete, i.e., there is a minimal distance between points of $\Gamma$, as 0 is an isolated point of $\Gamma-\Gamma$.

The FLC property of $\Gamma$ has the consequence that the orbit $\left\{t+\Gamma \mid t \in \mathbb{R}^{d}\right\}$ is precompact in the local topology (LT), where two point sets are close if, after a small translation, they agree on a large ball around $0 \in \mathbb{R}^{d}$. Consequently,

$$
\mathbb{X}(\Gamma):={\overline{\left\{t+\Gamma \mid t \in \mathbb{R}^{d}\right.}}^{\mathrm{LT}}
$$

is compact, and $\left(\mathbb{X}(\Gamma), \mathbb{R}^{d}\right)$ forms a topological dynamical system, on which one can define translation invariant probability measures, see [24] for details. The latter, at this point, need neither be unique nor ergodic. This can be seen by combining $2 \mathbb{Z}$ with an arbitrary (e.g., random) subset of $2 \mathbb{Z}+1$, which always produces an FLC set (and even a Meyer set, compare [20]). When using a standard Bernoulli process (e.g., coin tossing) to decide on the occupation of the sites from $2 \mathbb{Z}+1$, the hull $\mathbb{X}(\Gamma)$ almost surely contains the set $2 \mathbb{Z}$ as well as the set $\mathbb{Z}$ (since there are 0 - and 1-sequences of arbitrary length in almost all realizations of the Bernoulli process). Therefore different probability measures on $\mathbb{X}(\Gamma)$ clearly exist.

From now on, we restrict ourselves to FLC sets $\Gamma \subset \mathbb{R}^{d}$ such that $\left(\mathbb{X}(\Gamma), \mathbb{R}^{d}\right)$ is uniquely ergodic or even strictly ergodic, i.e., uniquely ergodic and minimal. In this setting, unique ergodicity is equivalent to $\Gamma$ having uniform cluster frequencies (UCF), and minimality to $\Gamma$ 
being repetitive, see [24] for details. This class contains lattices, but also systems obtained by primitive substitution rules [19] as well as regular generic model sets (also called cut and project sets) [21], hence two of the most important generalizations of lattices. Due to unique ergodicity, we then have a unique invariant probability measure $\vartheta$ on $\mathbb{X}(\Gamma)$, and $\left(\mathbb{X}(\Gamma), \mathbb{R}^{d}, \vartheta\right)$ is both a topological and a measurable dynamical system.

\subsection{A Result from Fourier Analysis}

Below, we need the following result from Fourier analysis concerning FLC sets.

Proposition 2.1 Let $\Gamma \subset \mathbb{R}^{d}$ be an FLC set, and consider the complex measure

$$
v:=\sum_{z \in \Gamma-\Gamma} g(z) \delta_{z}
$$

If $\sum_{z \in \Gamma-\Gamma}|g(z)|<\infty, v$ is a finite measure, and the Fourier transform $\hat{v}$ is a bounded, uniformly continuous function that defines an absolutely continuous measure on $\mathbb{R}^{d}$. Moreover, $\hat{v}$ is a generalized Fourier series,

$$
\hat{v}(k)=\sum_{z \in \Gamma-\Gamma} g(z) \mathrm{e}^{-2 \pi i k z}
$$

which converges uniformly and absolutely.

Proof Recall that a regular Borel measure $v$ is finite when $\|v\|=|v|\left(\mathbb{R}^{d}\right)<\infty$. Our $v$ is a pure point measure because $\Gamma-\Gamma$ is discrete and closed by the FLC property, so that $\|v\|=\sum_{z \in \Gamma-\Gamma}|g(z)|<\infty$ implies that $v$ is finite.

By the general properties of the Fourier (or Fourier-Stieltjes) transform, compare [23, Theorem 1.3.3], $\hat{v}$ is then a bounded and uniformly continuous function on $\mathbb{R}^{d}$. It is thus locally integrable, and hence defines an absolutely continuous measure on $\mathbb{R}^{d}$ by the RadonNikodym theorem.

The absolute (and hence also uniform) convergence of the generalized Fourier series follows from $\|v\|<\infty$ together with $\hat{\delta}_{z}(k)=\mathrm{e}^{-2 \pi i k z}$.

If $\Gamma-\Gamma$ is not only locally finite, but also uniformly discrete, one has the following explicit sufficient condition, which gives an easy access to the result of Proposition 2.1.

Lemma 2.2 Let $\Gamma \subset \mathbb{R}^{d}$ be an FLC set with $\Gamma-\Gamma$ uniformly discrete. If $g(z)$ is a complex function with

$$
g(z)=\mathcal{O}\left(|z|^{-d-\varepsilon}\right)
$$

for $|z| \rightarrow \infty$ and some $\varepsilon>0$, the measure $v=\sum_{z \in \Gamma-\Gamma} g(z) \delta_{z}$ is a finite measure, and Proposition 2.1 applies.

Proof $\Gamma-\Gamma$ uniformly discrete means that there is some $r>0$ so that any translate of the open ball $B_{r}(0)$ contains at most one point of $\Gamma-\Gamma$. Select a suitable lattice $L \subset \mathbb{R}^{d}$ so that $\mathbb{R}^{d}=L+B_{r}(0)$. For a lattice in $\mathbb{R}^{d}$, the sum

$$
\sum_{t \in L} \frac{1}{|t|^{d+\varepsilon}}
$$


is convergent. By standard arguments, one can now see that the sum $\sum_{z \in \Gamma-\Gamma} g(z)$ is absolutely convergent as a consequence of the assumed asymptotic properties. This gives $\|v\|<\infty$.

\subsection{A Result from the Theory of Gibbs Measures}

Let $\Gamma \subset \mathbb{R}^{d}$ be an FLC set with $\Gamma-\Gamma$ uniformly discrete. Put $\mathcal{S}:=\{F \subset \Gamma|0<| F \mid<\infty\}$ (the finite subsets of $\Gamma$, with $|F|:=\operatorname{card}(F)$ ) and consider an interaction potential $\left(\Phi_{S}\right)_{S \in \mathcal{S}}$ such that, for all $F \in \mathcal{S}$ and all $\omega \in\left\{c_{1}, \ldots, c_{n}\right\}^{\Gamma}$ with $c_{i} \in \mathbb{C}$, the total energy

$$
\mathcal{H}_{F}^{\Phi}(\omega)=\sum_{S \in \mathcal{S}, S \cap F \neq \varnothing} \Phi_{S}(\omega)
$$

of $\omega$ in $F$ for $\Phi$ exists. Let $\bar{F}=\Gamma \backslash F$ be the complement of $F$ and let $a b$ with $a \in$ $\left\{c_{1}, \ldots, c_{n}\right\}^{F}$ and $b \in\left\{c_{1}, \ldots, c_{n}\right\}^{\bar{F}}$ denote a combined configuration on $\Gamma$. In particular, we use this for $b=\omega_{\left.\right|_{\bar{F}}}$. The partition function for a finite subsystem $F$ is given by

$$
Z_{F}^{\Phi}(\omega)=\sum_{a \in\left\{c_{1}, \ldots, c_{n}\right\}^{F}} \mathrm{e}^{-\mathcal{H}_{F}^{\Phi}\left(a \omega_{\mid} \bar{F}\right.},
$$

and the probability measure

$$
A \mapsto \mu_{F}(A \mid \omega)=\frac{1}{Z_{F}^{\Phi}(\omega)} \sum_{a: a \omega_{\mid \bar{F}} \in A} \mathrm{e}^{-\mathcal{H}_{F}^{\Phi}\left(a \omega_{\mid}\right)},
$$

for all $A \in \mathcal{A}$ (the product $\sigma$-algebra on $\left\{c_{1}, \ldots, c_{n}\right\}^{\Gamma}$ ), is called the Gibbs distribution in $F$ with boundary condition $\omega_{\left.\right|_{\bar{F}}}$ and interaction potential $\Phi$, and defines the associated Gibbs measure $\mu$, see [11] for details.

We now show that, on one essential condition, the covariance of $\Omega_{x}:\left\{c_{1}, \ldots, c_{n}\right\}^{\Gamma} \rightarrow$ $\left\{c_{1}, \ldots, c_{n}\right\}, \omega \mapsto \omega_{x}$ and $\overline{\Omega_{y}}:\left\{c_{1}, \ldots, c_{n}\right\}^{\Gamma} \rightarrow\left\{\overline{c_{1}}, \ldots, \overline{c_{n}}\right\}, \omega \mapsto \overline{\omega_{y}}$ is summable in $y$, and that the sums are bounded for all $x$.

Proposition 2.3 Consider the FLC set $\Gamma$ and any metric $\mathrm{d}(x, y)$. For $S \in \mathcal{S}$, let $\operatorname{diam}(S)$ denote the associated diameter of $S$, and define

$$
\mathcal{D}\left(\Phi_{S}\right)=\sup _{\zeta, \lambda \in\left\{c_{1}, \ldots, c_{n}\right\}^{\Gamma}}\left|\Phi_{S}(\zeta)-\Phi_{S}(\lambda)\right|
$$

On the condition that

$$
\sup _{u \in \Gamma} \sum_{u \in S \in \mathcal{S}} \mathrm{e}^{\operatorname{diam}(S)}(|S|-1) \mathcal{D}\left(\Phi_{S}\right)<2,
$$

the corresponding Gibbs measure $\mu$ is unique. Moreover, for all $x \in \Gamma$,

$$
\sum_{y \in \Gamma}\left|\operatorname{cov}_{\mu}\left(\Omega_{x}, \overline{\Omega_{y}}\right)\right|<c .
$$

Proof Let $\|$.$\| be the variation norm. Dobrushin's interaction matrix C=\left(C_{u v}\right)(u, v \in \Gamma)$ describes the dependence between the particles at different sites and is defined by

$$
C_{u v}=\sup \left\{\left\|\mu_{\{u\}}(\cdot \mid \zeta)-\mu_{\{u\}}(\cdot \mid \lambda)\right\| \mid \zeta=\lambda \text { off } v\right\}
$$


Thus, these elements quantify the largest variational distance between the conditional distributions $\mathrm{d} \mu_{\{u\}}\left(\omega_{\mid u} \mid \omega\right)$ for a fixed site $u$ evaluated at two configurations which differ from each other only at site $v$.

In the proof of [11, Proposition 8.8], it was shown that

$$
C_{u v} \leq \frac{1}{2} \sum_{\{u, v\} \subset S \in \mathcal{S}} \mathcal{D}\left(\Phi_{S}\right)
$$

Therefore, due to (1),

$$
\begin{aligned}
\alpha & :=\sup _{u} \sum_{v} \mathrm{e}^{\mathrm{d}(u, v)} C_{u v} \leq \frac{1}{2} \sup _{u} \sum_{v \neq u} \sum_{S \supset\{u, v\}} \mathcal{D}\left(\Phi_{S}\right) \mathrm{e}^{\mathrm{d}(u, v)} \\
& \leq \frac{1}{2} \sup _{u} \sum_{u \in S \in \mathcal{S}} \mathrm{e}^{\operatorname{diam}(S)}(|S|-1) \mathcal{D}\left(\Phi_{S}\right)<1
\end{aligned}
$$

holds (which implies uniqueness of the Gibbs measure because $\sup _{u} \sum_{v} C_{u v}<1$ ), and we may apply [11, Proposition 8.34] which states that

$$
\left|\operatorname{cov}_{\mu}(f, g)\right| \leq \frac{1}{4} \sum_{u, v \in \Gamma} \mathcal{D}_{u}(f) D_{u v} \mathcal{D}_{v}(g)
$$

where $f$ and $g$ are bounded quasilocal functions on $\left\{c_{1}, \ldots, c_{n}\right\}^{\Gamma}$,

$$
\mathcal{D}_{u}(f)=\sup \{|f(\zeta)-f(\lambda)| \mid \zeta=\lambda \text { off } u\}
$$

and $D_{u v}=\sum_{n=0}^{\infty} C_{u v}^{n}$. Recall that a measurable function is called quasilocal when

$$
\lim _{F \in \mathcal{S}} \sup _{\substack{\zeta,\left.\lambda \in\left\{c_{1}, \ldots, c_{n}\right\} \\ \zeta\right|_{F}=\lambda_{F}}}|f(\zeta)-f(\lambda)|=0
$$

Here, the notation $\lim _{F \in \mathcal{S}}$ means that the limit is taken along sets, where more and more points are added, so that $|F| \nearrow \infty$.

Let $f(\omega)=\omega_{x}$ and $g(\omega)=\overline{\omega_{y}}$. This results in

$$
\mathcal{D}_{u}(f)= \begin{cases}\sup _{i, j}\left|c_{i}-c_{j}\right|, & \text { if } u=x \\ 0, & \text { otherwise }\end{cases}
$$

and

$$
\mathcal{D}_{v}(g)= \begin{cases}\sup _{i, j}\left|c_{i}-c_{j}\right|, & \text { if } v=y \\ 0, & \text { otherwise. }\end{cases}
$$

Then, (2) implies

$$
\sum_{y \in \Gamma}\left|\operatorname{cov}_{\mu}\left(\Omega_{x}, \overline{\Omega_{y}}\right)\right| \leq \sum_{y \in \Gamma} \frac{\left(\sup _{i, j}\left|c_{i}-c_{j}\right|\right)^{2}}{4} D_{x y} \leq \frac{\left(\sup _{i, j}\left|c_{i}-c_{j}\right|\right)^{2}}{4(1-\alpha)}=c,
$$

since [11, Remark 8.26] states that $\sum_{y \in \Gamma} D_{x y} \leq 1 /(1-\alpha)$. Moreover, $c$ does not depend on $x$, wherefore we get the result. 


\section{Diffraction Theory}

The diffraction measure of a solid describes the outcome of a kinematic diffraction experiment, e.g., by $X$-rays. It is the Fourier transform of the autocorrelation measure of the solid, see [13] for the underlying theory in terms of translation bounded (complex) measures and $[9,12]$ for general background on the physics of diffraction.

\subsection{Diffraction of a Perfect Lattice}

If we start with a perfect lattice $\Gamma$ in Euclidean space (i.e., a co-compact discrete subgroup of $\mathbb{R}^{d}$ ) containing 0, its Dirac comb $\delta_{\Gamma}:=\sum_{z \in \Gamma} \delta_{z}$ is a model for the atomic positions (of a mono-atomic crystal, say). In this case, the autocorrelation is simply given by

$$
\gamma=\sum_{z \in \Gamma} \eta(z) \delta_{z}
$$

with the autocorrelation coefficients

$$
\eta(z):=\lim _{r \rightarrow \infty} \frac{1}{\operatorname{vol}\left(B_{r}\right)} \operatorname{card}\left(\Gamma_{r} \cap\left(z+\Gamma_{r}\right)\right)
$$

where $\Gamma_{r}:=\Gamma \cap B_{r}(0)$ with $B_{r}(0)$ the open ball of radius $r$ centred at 0 . Clearly, $\eta(z)=$ $\operatorname{dens}(\Gamma)$ for all $z \in \Gamma$, so that $\gamma=\operatorname{dens}(\Gamma) \cdot \delta_{\Gamma}$. Observing Poisson's summation formula for Dirac combs [25, (VII.7.4)],

$$
\hat{\delta}_{\Gamma}=\operatorname{dens}(\Gamma) \cdot \delta_{\Gamma^{*}}
$$

with the dual lattice $\Gamma^{*}$, one obtains the well-known formula

$$
\hat{\gamma}=(\operatorname{dens}(\Gamma))^{2} \cdot \delta_{\Gamma^{*}}
$$

for the diffraction from a perfect lattice.

This can easily be generalized to any measure of the form

$$
v=\rho * \delta_{\Gamma}
$$

with $\rho$ some finite measure, which is well-defined, compare [8, Proposition 1.13]. The diffraction then gives

$$
\hat{\gamma}_{\nu}=|\hat{\rho}|^{2} \cdot(\operatorname{dens}(\Gamma))^{2} \cdot \delta_{\Gamma^{*}}
$$

by an application of the convolution theorem. The finite measure $\rho$ can accommodate the distribution of finitely many possibly different atoms over the unit cell of $\Gamma$ as well as characteristic profiles of the atoms. The result shows up as the continuous modulation factor $|\hat{\rho}|^{2}$ in the diffraction measure, see [9] for various applications.

\subsection{Diffraction of Lattice Gases}

We now turn to the rather general situation of a lattice $\Gamma$ occupied with $n \in \mathbb{N}$ different types of scatterers with scattering strengths $\left\{c_{1}, \ldots, c_{n}\right\}, c_{i} \in \mathbb{C}$, subject to some stochastic process in equilibrium. Let $\left(H_{x}\right)_{x \in \Gamma}$ be a family of random variables describing the scattering strengths at the lattice positions. Since each $H_{x}$ takes one of finitely many finite values, 
$h_{x} \in\left\{c_{1}, \ldots, c_{n}\right\}$, the second moments of the random variables exist. Moreover, we assume that the family of random variables is controlled by a translation invariant Gibbs measure $\mu$. Furthermore, we suppose that $\mu$ is ergodic. The natural autocorrelation, if it exists, is given by

$$
\gamma^{(H)}=\sum_{z \in \Gamma} \eta^{(H)}(z) \delta_{z}
$$

where, with $\Gamma_{r}=\Gamma \cap B_{r}$,

$$
\eta^{(H)}(z):=\lim _{r \rightarrow \infty} \frac{1}{\operatorname{vol}\left(B_{r}\right)} \sum_{x \in \Gamma_{r}} H_{x} \overline{H_{x-z}} .
$$

Lemma 3.1 With the above-mentioned assumptions, the equation

$$
\gamma^{(H)}=\left|\mathbb{E}_{\mu}\left(H_{0}\right)\right|^{2} \gamma+\operatorname{dens}(\Gamma) \sum_{z \in \Gamma} \operatorname{cov}_{\mu}\left(H_{0}, \overline{H_{-z}}\right) \delta_{z}
$$

holds $\mu$-a.s., where $\gamma$ is the autocorrelation of the fully occupied lattice and one has $\operatorname{cov}_{\mu}\left(H_{0}, \overline{H_{-z}}\right)=\mathbb{E}_{\mu}\left(H_{0} \overline{H_{-z}}\right)-\left|\mathbb{E}_{\mu}\left(H_{0}\right)\right|^{2}$.

Proof Let $T_{x}$ denote the shift map, i.e., let $T_{x}\left(H_{0} \overline{H_{-z}}\right):=H_{x} \overline{H_{x-z}}$. Due to Birkhoff's ergodic theorem [16, Chapter 2], one has $\mu$-a.s.:

$$
\begin{aligned}
\eta^{(H)}(z) & =\lim _{r \rightarrow \infty} \frac{1}{\operatorname{vol}\left(B_{r}\right)} \sum_{x \in \Gamma_{r}} T_{x}\left(H_{0} \overline{H_{-z}}\right)=\lim _{r \rightarrow \infty} \frac{\operatorname{card}\left(\Gamma_{r}\right)}{\operatorname{vol}\left(B_{r}\right)} \mathbb{E}_{\mu}\left(H_{0} \overline{H_{-z}}\right) \\
& =\operatorname{dens}(\Gamma) \mathbb{E}_{\mu}\left(H_{0} \overline{H_{-z}}\right) .
\end{aligned}
$$

Then, by using the fact that $\mathbb{E}_{\mu}\left(\overline{H_{-z}}\right)=\mathbb{E}_{\mu}\left(\overline{H_{0}}\right)$ for all $z \in \Gamma$, one has

$$
\begin{aligned}
\gamma^{(H)} & =\sum_{z \in \Gamma} \operatorname{dens}(\Gamma) \mathbb{E}_{\mu}\left(H_{0} \overline{H_{-z}}\right) \delta_{z} \\
& =\sum_{z \in \Gamma} \operatorname{dens}(\Gamma)\left|\mathbb{E}_{\mu}\left(H_{0}\right)\right|^{2} \delta_{z}+\sum_{z \in \Gamma} \operatorname{dens}(\Gamma)\left(\mathbb{E}_{\mu}\left(H_{0} \overline{H_{-z}}\right)-\left|\mathbb{E}_{\mu}\left(H_{0}\right)\right|^{2}\right) \delta_{z} \\
& =\left|\mathbb{E}_{\mu}\left(H_{0}\right)\right|^{2} \gamma+\operatorname{dens}(\Gamma) \sum_{z \in \Gamma} \operatorname{cov}_{\mu}\left(H_{0}, \overline{H_{-z}}\right) \delta_{z},
\end{aligned}
$$

which establishes the claim.

Note that the splitting in (5) makes sense in terms of diffraction theory whenever the second term is well-behaved (e.g., when the covariance falls sufficiently rapidly).

\section{Special Lattice Gases}

In what follows, we analyse the diffraction spectra of lattice gases as described in the previous section, subject to some local or short-range interaction of stochastic nature. Here, we use $\Gamma=\mathbb{Z}^{d}$ for simplicity, but the results do not really depend on this particular choice of a lattice. In fact, they are robust under non-singular affine transformations. We show that, on 
one essential condition, the diffraction spectrum of such a lattice gas model does not contain a singular continuous part.

\section{Theorem 4.1 On the condition that}

$$
\sup _{x \in \mathbb{Z}^{d}} \sum_{x \in S \in \mathcal{S}} \mathrm{e}^{\operatorname{diam}(S)}(|S|-1) \mathcal{D}\left(\Phi_{S}\right)<2,
$$

the corresponding Gibbs measure $\mu$ is unique. Moreover, the diffraction spectrum of the corresponding lattice gas model with $n$ different types of particles $\mu$-a.s. exists, is $\mathbb{Z}^{d}$-periodic and consists of a pure point part and an absolutely continuous part with continuous density. No singular continuous part is present.

Proof In the present case, as in Lemma 3.1, the autocorrelation measure is given by

$$
\gamma^{(H)}=\sum_{x \in \mathbb{Z}^{d}} \mathbb{E}_{\mu}\left(H_{0}, \overline{H_{-x}}\right) \delta_{x}=\left|\mathbb{E}_{\mu}\left(H_{0}\right)\right|^{2} \delta_{\mathbb{Z}^{d}}+\sum_{x \in \mathbb{Z}^{d}} \operatorname{cov}_{\mu}\left(H_{0}, \overline{H_{-x}}\right) \delta_{x} .
$$

This holds for $\mu$-almost all elements of our lattice gas ensemble (respectively for $\mu$-almost all realizations of the corresponding stochastic process).

The first part of the autocorrelation gives, under Fourier transform, the pure point part of the diffraction measure, by means of the Poisson summation formula (3) for lattice Dirac combs (compare [6] and references given there). This part is clearly $\mathbb{Z}^{d}$-periodic.

Furthermore, due to Proposition 2.3, we have $\sum_{x \in \mathbb{Z}^{d}}\left|\operatorname{cov}_{\mu}\left(H_{0}, \overline{H_{-x}}\right)\right|<\infty$. Therefore, by Proposition 2.1 with $\Gamma-\Gamma=\mathbb{Z}^{d}$, the second part of the autocorrelation, under explicit Fourier transform according to Proposition 2.1, gives the absolutely continuous part of the diffraction measure. It is again $\mathbb{Z}^{d}$-periodic (compare [1,6] for a more general explanation of this phenomenon), and our claim follows.

Let us look at the implications of Theorem 4.1 for some special models that are relevant to crystallographic applications.

First, we consider finite range potentials $\left(\Phi_{S}\right)_{S \in \mathcal{S}}=\left(\beta \Phi_{S}^{*}\right)_{S \in \mathcal{S}}$ with $\beta:=1 /\left(k_{B} T\right)$ (the inverse temperature). This means that

$$
\sup \left\{\operatorname{diam}(S) \mid S \in \mathcal{S}, \Phi_{S} \neq 0\right\}=R<\infty,
$$

where $R$ denotes the range. For sufficiently small $\beta$, this implies

$$
\sup _{x} \sum_{x \in S \in \mathcal{S}} \mathrm{e}^{\operatorname{diam}(S)}(|S|-1) \mathcal{D}\left(\Phi_{S}\right) \leq \mathrm{e}^{R} \sup _{x} \sum_{x \in S \in \mathcal{S}}(|S|-1) \mathcal{D}\left(\Phi_{S}\right) \leq c \beta<2,
$$

where $c$ is a constant. Indeed, the finite number of $S \ni x$ with $\Phi_{S} \neq 0$ results in a finite sum. Moreover, because of the finite range, there is a maximum of $|S|$ and $\mathcal{D}\left(\Phi_{S}\right)$ for all $S$ with $\operatorname{diam}(S) \leq R$. Thus, for sufficiently high temperatures, Condition (1) holds and, according to Theorem 4.1, the diffraction spectrum of such a model contains no singular continuous part for such temperatures. This is most likely true in more generality, but does not follow from our simplified approach.

Remark 1 A well-known model of this type is the Potts model, where only pair interactions of nearest neighbors occur. This includes the classic ferromagnetic Ising model, where absence of singular continuous diffraction is already known for all temperatures, including the critical point $[4,6]$. 
Now, we consider potentials with exponentially or algebraically decaying pair interactions. Thus, the interaction potential is given by

$$
\Phi_{S}(\omega)= \begin{cases}\beta \phi\left(\omega_{\mid x, y}\right) J(x-y), & \text { if } S=\{x, y\} \\ 0, & \text { otherwise }\end{cases}
$$

with $\phi:\left\{c_{1}, \ldots, c_{n}\right\} \times\left\{c_{1}, \ldots, c_{n}\right\} \rightarrow \mathbb{C}$, and we suppose that we have either

$$
\sum_{x \in \mathbb{Z}^{d}} \mathrm{e}^{t\|x\|}|J(x)|<\infty
$$

or

$$
\sum_{x \in \mathbb{Z}^{d}}\|x\|^{p}|J(x)|<\infty
$$

for some positive constants $t$ and $p>1$. Then, for sufficiently high temperatures, the diffraction spectrum for such a model contains no singular continuous part.

Argument Consider the metric $\mathrm{d}(x, y):=t\|x-y\| \wedge\lfloor p\rfloor \log (1+\|x-y\|)$ with some constants $t>0$ and $p>1$, where $a \wedge b$ means the minimum of $a$ and $b$. It is not difficult to check that this is indeed a metric. Then, (7) results, for sufficiently high temperatures, in

$$
\sup _{x} \sum_{x \in S \in \mathcal{S}} \mathrm{e}^{\operatorname{diam}(S)}(|S|-1) \mathcal{D}\left(\Phi_{S}\right) \leq \beta \sup \left(\left|\phi\left(\zeta_{\mid x, 0}\right)-\phi\left(\lambda_{\mid x, 0}\right)\right|\right) \sum_{x \in \mathbb{Z}^{d}} \mathrm{e}^{t\|x\|}|J(x)|<2
$$

and, again for sufficiently high temperatures, (8) results in the estimate

$$
\sup _{x} \sum_{x \in S \in \mathcal{S}} \mathrm{e}^{\operatorname{diam}(S)}(|S|-1) \mathcal{D}\left(\Phi_{S}\right) \leq c \beta \sup \left(\left|\phi\left(\zeta_{\mid x, 0}\right)-\phi\left(\lambda_{\left.\right|_{x, 0}}\right)\right|\right) \sum_{x \in \mathbb{Z}^{d}}\|x\|^{p}|J(x)|<2 .
$$

Note that, in addition to $\operatorname{diam}(S) \leq t\|x-y\|$, one has $\operatorname{diam}(S) \leq\lfloor p\rfloor \log (1+\|x-y\|)$ in this metric, which implies the estimate

$$
\mathrm{e}^{\operatorname{diam}(S)} \leq(1+\|x\|)^{\lfloor p\rfloor}=\sum_{k=0}^{\lfloor p\rfloor}\left(\begin{array}{c}
\lfloor p\rfloor \\
k
\end{array}\right)\|x\|^{\lfloor p\rfloor} \leq \sum_{k=0}^{\lfloor p\rfloor}\left(\begin{array}{c}
\lfloor p\rfloor \\
k
\end{array}\right)\|x\|^{p}=c\|x\|^{p} .
$$

Thus, applying Theorem 4.1 once more, we obtain the absence of singular continuous diffraction for all sufficiently high temperatures.

\section{Extension to More General FLC Sets}

The result of Theorem 4.1 is inherently robust and one would expect analogous results beyond the lattice case. Of particular interest (e.g., in the mathematical theory of quasicrystals) is the general class of point sets of finite local complexity, as introduced in Sect. 2.

\subsection{Diffraction without Disorder}

In our setting with $\Gamma$ an FLC set and $\left(\mathbb{X}(\Gamma), \mathbb{R}^{d}\right)$ uniquely ergodic, each element $\Gamma^{\prime} \in \mathbb{X}(\Gamma)$ is itself an FLC set. (Note that, due to the fact that $\Gamma-\Gamma$ is locally finite, any element of 
$\mathbb{X}(\Gamma)$ can at most possess clusters that also occur in $\Gamma$.) The corresponding Dirac comb $\delta_{\Gamma^{\prime}}$ is then a translation bounded (and thus tempered) measure whose autocorrelation

$$
\gamma_{\Gamma^{\prime}}:=\lim _{r \rightarrow \infty} \frac{1}{\operatorname{vol}\left(B_{r}\right)} \delta_{\Gamma^{\prime} \cap B_{r}} *\left(\delta_{\Gamma^{\prime} \cap B_{r}}\right)^{\sim}
$$

exists, where $\tilde{v}$ is the measure defined by $\tilde{v}(g)=\overline{v(\tilde{g})}$ with $\tilde{g}(x):=\overline{g(-x)}$, see $[13,24]$ for details.

Proposition 5.1 Let $\Gamma$ be an FLC set such that $\left(\mathbb{X}(\Gamma), \mathbb{R}^{d}\right)$ is uniquely ergodic. Then, each $\Gamma^{\prime} \in \mathbb{X}(\Gamma)$ has the same autocorrelation $\gamma$. The latter is a positive and positive definite pure point measure that can be written as

$$
\gamma=\sum_{z \in \Gamma-\Gamma} \eta(z) \delta_{z}
$$

with the autocorrelation coefficients

$$
\eta(z):=\lim _{r \rightarrow \infty} \frac{1}{\operatorname{vol}\left(B_{r}\right)} \operatorname{card}\left(\Gamma_{r} \cap\left(z+\Gamma_{r}\right)\right) .
$$

Proof By definition of $\mathbb{X}(\Gamma)$, each $\Gamma^{\prime} \in \mathbb{X}(\Gamma)$ is the limit of some sequence of the form $\left(t_{n}+\Gamma\right)$ in the local topology. Clearly, for any $t \in \mathbb{R}^{d}, \Gamma$ and $t+\Gamma$ possess the same autocorrelation, i.e., $\gamma_{t+\Gamma}=\gamma_{\Gamma}$. Moreover, unique ergodicity implies that

$$
\gamma_{t+\Gamma}^{(r)}:=\frac{1}{\operatorname{vol}\left(B_{r}\right)} \delta_{(t+\Gamma) \cap B_{r}} *\left(\delta_{(t+\Gamma) \cap B_{r}}\right)^{\sim}=\gamma_{\Gamma}^{(r)}+o(1), \quad \text { as } r \rightarrow \infty,
$$

where the $o(1)$ term is uniform in $t$ (due to UCF, see Sect. 2).

Then, when considering $\gamma_{t_{n}+\Gamma}^{(r)}$, the two limits $r \rightarrow \infty$ and $n \rightarrow \infty$ commute, which is tantamount to saying that the mapping $\Gamma^{\prime} \mapsto \gamma_{\Gamma^{\prime}}$ is continuous on $\mathbb{X}(\Gamma)$. Since $\gamma_{t_{n}+\Gamma} \equiv \gamma_{\Gamma}$, this means that $\gamma_{\Gamma^{\prime}} \equiv \gamma_{\Gamma}=\gamma$ on $\mathbb{X}(\Gamma)$.

Translation boundedness, positivity and positive definiteness are standard and clear by construction, while the explicit representation of $\gamma$ as a pure point measure is a consequence of $\Gamma-\Gamma$ being locally finite. The formula for $\eta(z)$ is a simple calculation, the limit exists due to unique ergodicity, compare [5] for details and for an alternative approach via the unique ergodic measure $\vartheta$ on $\mathbb{X}(\Gamma)$.

Corollary 5.2 Under the assumptions of Proposition 5.1, also the diffraction measure $\hat{\gamma}$ is the same for all elements of the hull $\mathbb{X}(\Gamma)$. It is a positive, translation bounded measure on $\mathbb{R}^{d}$, with a unique decomposition

$$
\hat{\gamma}=\hat{\gamma}_{\mathrm{pp}}+\hat{\gamma}_{\mathrm{sc}}+\hat{\gamma}_{\mathrm{ac}}
$$

into its pure point, singular continuous and absolutely continuous parts, relative to Lebesgue measure as reference (being the Haar measure of $\mathbb{R}^{d}$ ).

This generalizes the setting of crystallographic sets, the latter being examples of pure point diffractive systems. Other examples with pure point diffraction are regular model sets, also called cut and project sets [21]. In general, however, other spectral types, or mixtures, can occur $[4,14,15]$. 


\subsection{Influence of Disorder}

Let us first look at an interaction-free particle gas on $\Gamma$, where we continue to assume that $\Gamma$ is FLC with $\left(\mathbb{X}(\Gamma), \mathbb{R}^{d}\right)$ uniquely ergodic. Let $\vartheta$ be the unique translation invariant probability measure on $\mathbb{X}(\Gamma)$. To describe the system, we consider an i.i.d. family of random variables, labeled by the points of $\Gamma$. For simplicity, we assume that each variable $H_{x}$ takes a (complex) value $h_{x} \in\left\{c_{1}, \ldots, c_{n}\right\}$, with attached probabilities $p_{1}, \ldots, p_{n}$.

In line with Corollary 5.2, let $\hat{\gamma}$ denote the diffraction measure of $\Gamma$. By standard arguments $[3,4,17,18]$, one then obtains the following result for the diffraction of the particle gas, where the $c_{i}$ serve as weights that represent the scattering strength at the corresponding point of $\Gamma$.

Theorem 5.3 Let $\delta_{\Gamma}^{(H)}:=\sum_{x \in \Gamma} H_{x} \delta_{x}$ be a Bernoulli process on $\Gamma$ with i.i.d. random variables $H_{x}$ that take values $h_{x} \in\left\{c_{1}, \ldots, c_{n}\right\}$, according to a common probability vector $p$, and assume that $0 \in \Gamma$.

Then, the autocorrelation $\gamma^{(H)}$ almost surely exists and results in the diffraction measure

$$
\hat{\gamma}^{(H)}=\left|\mathbb{E}_{p}\left(H_{0}\right)\right|^{2} \hat{\gamma}+\operatorname{dens}(\Gamma)\left(\mathbb{E}_{p}\left(\left|H_{0}\right|^{2}\right)-\left|\mathbb{E}_{p}\left(H_{0}\right)\right|^{2}\right),
$$

where the second term on the right hand side is a constant and hence a contribution to the absolutely continuous part of $\hat{\gamma}^{(H)}$.

The key in proving this result is to establish that the new autocorrelation is still concentrated on $\Gamma-\Gamma$, with coefficients

$$
\eta^{(H)}(z)=\left|\mathbb{E}_{p}\left(H_{0}\right)\right|^{2} \eta(z)+\operatorname{dens}(\Gamma)\left(\mathbb{E}_{p}\left(\left|H_{0}\right|^{2}\right)-\left|\mathbb{E}_{p}\left(H_{0}\right)\right|^{2}\right) \delta_{z, 0}
$$

with $\mathbb{E}_{p}\left(H_{0}\right)=\sum_{i=1}^{n} p_{i} c_{i}$ etc. Due to the i.i.d. nature, the validity of this type of result can be extended to more general systems, compare [17, 18].

We now begin to extend our analysis to more interesting types of disorder. Instead of an i.i.d. family of random variables, we consider a family of random variables which are controlled by a Gibbs measure $\mu_{\Gamma}$. Moreover, we have the unique ergodic measure $\vartheta$ on $\mathbb{X}(\Gamma)$ that is essentially defined by the cluster frequencies $f_{\mathfrak{y}}$ for arbitrary clusters $\mathfrak{y}$. In the following, we consider the measures

$$
\delta_{\Gamma}=\sum_{x \in \Gamma} \delta_{x}, \quad \delta_{\Gamma}^{(H)}=\sum_{x \in \Gamma} H_{x} \delta_{x} \quad \text { and } \quad \mathbb{E}_{\mu_{\Gamma}}\left(\delta_{\Gamma}^{(H)}\right)=\sum_{x \in \Gamma} \mathbb{E}_{\mu_{\Gamma}}\left(H_{x}\right) \delta_{x} .
$$

Moreover, we define $\mathbb{X}\left(\delta_{\Gamma}\right)$ as the orbit closure of $\delta_{\Gamma}$ in the vague topology. Note that there is a topological conjugacy between $(\Gamma, \mathbb{X}(\Gamma))$ and $\left(\delta_{\Gamma}, \mathbb{X}\left(\delta_{\Gamma}\right)\right)$, see [5, Lemma 2].

Theorem 5.4 Assume that $\delta_{\Gamma}$ has a pure point diffraction spectrum. On the three conditions that the map $\mathbb{X}\left(\delta_{\Gamma}\right) \ni \delta_{\Gamma^{\prime}} \mapsto \mathbb{E}_{\mu_{\Gamma^{\prime}}}\left(\delta_{\Gamma^{\prime}}^{(H)}\right)$ is continuous, that the natural autocorrelations of $\delta_{\Gamma}^{(H)}$ and $\mathbb{E}_{\mu_{\Gamma}}\left(\delta_{\Gamma}^{(H)}\right)$ a.s. exist and that the difference between the autocorrelation coefficients is summable, the diffraction spectrum of the particle gas with $n$ different types of particles consists of a pure point part and an absolutely continuous part with continuous density. No singular continuous part is present. 
Proof $\left(\mathbb{X}\left(\mathbb{E}_{\mu_{\Gamma}}\left(\delta_{\Gamma}^{(H)}\right)\right), \mathbb{R}^{d}\right)$ is a factor of $\left(\mathbb{X}\left(\delta_{\Gamma}\right), \mathbb{R}^{d}\right)$. Since $\delta_{\Gamma}$ has a pure point diffraction spectrum by assumption, this is inherited by $\mathbb{E}_{\mu_{\Gamma}}\left(\delta_{\Gamma}^{(H)}\right)$ due to [7, Proposition 1 and Theorem 2]. Moreover, the difference between the autocorrelation coefficients is summable and therefore only results in an absolutely continuous part of the diffraction spectrum.

Corollary 5.5 In the case of a regular model set, finite range potential and sufficiently high temperatures, there is no singular continuous part present in the diffraction spectrum of the particle gas on this set.

Proof Continuity of the map $\mathbb{X}\left(\delta_{\Gamma}\right) \ni \delta_{\Gamma^{\prime}} \mapsto \mathbb{E}_{\mu_{\Gamma^{\prime}}}\left(\delta_{\Gamma^{\prime}}^{(H)}\right)$ is obvious due to the finite range potential. Let $\mathfrak{Y}_{|z|}:=$ clusters of radius $|z|+R$ around 0$\}$ be the set of all clusters with specified radius, where $R$ is the range of the potential. A regular model set has uniform cluster frequencies $f_{\mathfrak{y}}$ (chosen such that $\sum_{\mathfrak{y} \in \mathfrak{Y}_{|z|}} f_{\mathfrak{y}}=1$ ), see [24, Theorem 4.5]. Together with the finite range potential this is the reason why we may use the strong law of large numbers and get

$$
\begin{aligned}
\eta^{(H)}(z)= & \lim _{r \rightarrow \infty} \frac{1}{\operatorname{vol}\left(B_{r}\right)} \sum_{x \in \Gamma_{r}} H_{x} \overline{H_{x-z}} \\
= & \lim _{r \rightarrow \infty} \frac{\operatorname{card}\left(\Gamma_{r}\right)}{\operatorname{vol}\left(B_{r}\right)} \sum_{\mathfrak{y} \in \mathfrak{Y}_{|z|}} \frac{1}{\operatorname{card}\left(\Gamma_{r}\right)} \sum_{\substack{x \in \Gamma_{r} \\
(\Gamma-x) \cap B_{|z|+R}=\mathfrak{y}}} H_{x} \overline{H_{x-z}} \\
= & \operatorname{dens}(\Gamma) \sum_{\mathfrak{y} \in \mathfrak{Y}_{|z|}} f_{\mathfrak{y}} \mathbb{E}_{\mu_{\Gamma}}\left(H_{x} \overline{H_{x-z}} \mid(\Gamma-x) \cap B_{|z|+R}=\mathfrak{y}\right) \\
= & \operatorname{dens}(\Gamma) \sum_{\mathfrak{y} \in \mathfrak{Y}_{|z|}} f_{\mathfrak{y}} \mathbb{E}_{\mu_{\Gamma}}\left(H_{x} \mid(\Gamma-x) \cap B_{|z|+R}=\mathfrak{y}\right) \\
& \times \mathbb{E}_{\mu_{\Gamma}}\left(\overline{H_{x-z}} \mid(\Gamma-\Gamma) \cap B_{|z|+R}=\mathfrak{y}\right) \\
& +\operatorname{dens}(\Gamma) \sum_{\mathfrak{y} \in \mathfrak{Y}_{|z|}} f_{\mathfrak{y}} \operatorname{cov}_{\mu_{\Gamma}}\left(H_{x} \overline{H_{x-z} \mid}(\Gamma-x) \cap B_{|z|+R}=\mathfrak{y}\right) \\
= & \lim _{r \rightarrow \infty} \frac{1}{\operatorname{vol}\left(B_{r}\right)} \sum_{x \in \Gamma_{r}} \mathbb{E}_{\mu_{\Gamma}}\left(H_{x}\right) \mathbb{E}_{\mu_{\Gamma}}\left(\overline{H_{x-z}}\right) \\
& +\operatorname{dens}(\Gamma) \sum_{\mathfrak{y} \in \mathfrak{Y}_{|z|}} f_{\mathfrak{y}} \operatorname{cov}_{\mu_{\Gamma}}\left(H_{x} \overline{H_{x-z} \mid}(\Gamma-x) \cap B_{|z|+R}=\mathfrak{y}\right) .
\end{aligned}
$$

Note that, in order to apply the strong law of large numbers, we first have to split the last sum in the first line into a finite number of sums over points with non-overlapping surroundings of radius $|z|+R$. The law of large numbers is then applied to each sum separately, each of which a.s. converges to the same limit, compare $[2,13,22]$ for related results.

In the end, the first term is the autocorrelation coefficient of the averaged Dirac comb $\mathbb{E}_{\mu_{\Gamma}}\left(\delta_{\Gamma}^{(H)}\right)$ at $z$. Along the lines of Sect. 4, one can show that condition (1) holds. Therefore, due to Proposition 2.3, $\sum_{z \in \Gamma-\Gamma} \operatorname{dens}(\Gamma) \sum_{\mathfrak{y} \in \mathfrak{Y}|z|} f_{\mathfrak{y}}\left|\operatorname{cov}_{\mu_{\Gamma}}\left(H_{x} \overline{H_{x-z}} \mid(\Gamma-x) \cap B_{|z|+R}=\mathfrak{y}\right)\right|$ is finite. Moreover, the diffraction of a regular model set is pure point (compare [24, Theorem 4.5]). Therefore, from Theorem 5.4, we obtain the absence of a singular continuous part in the diffraction spectrum of the particle gas. 
Remark 2 In general, a singular continuous diffraction component of a particle gas on a graph in $\mathbb{R}^{d}$ seems possible due to two sources. On the one hand, it could be a peculiar longrange structure or irregularity of the graph, which is excluded by the model set assumption in our case. On the other hand, it could be caused by some long-range order in the Gibbs or ground state measure, which is excluded by range condition together with the temperature assumption that leads to a Dobrushin uniqueness regime.

\section{Outlook}

The results of this paper, taken together with those of $[17,18]$, demonstrate that many results known from the theory of lattice gases can be extended to more general types of systems. In particular, model sets behave in very much the same way as crystals to the superposition of stochastic disorder. This is also the case in related studies that employ point process methods to derive explicit examples, compare [2] and references therein.

The conditions investigated here for the non-periodic systems are to be considered as a first step. It is quite clear that the condition of finite range in Corollary 5.5 can be lifted, but the proofs will then become technically more involved. This calls for an alternative or modified point of view to simplify the approach.

In this paper, we have considered FLC sets together with configuration spaces based on finite type spaces. It is clear that one can now also increase the generality towards countably many types per site, or even to continuous type spaces. This certainly adds another layer of technical complication, but we expect the principal results to remain unchanged.

Acknowledgements It is our pleasure to thank H.-O. Georgii, Yu. Kondratiev and M. Röckner for discussions and useful suggestions as well as K. Matzutt for useful hints on the manuscript. We also thank an anonymous referee for a number of valuable comments and questions. This work was supported by the German Research Council (DFG), within the CRC 701.

\section{References}

1. Baake, M.: Diffraction of weighted lattice subsets. Can. Math. Bull. 45, 483-498 (2002), arXiv: math.MG/0106111

2. Baake, M., Birkner, M., Moody, R.V.: Diffraction of stochastic point sets: exactly solvable examples. In preparation (2007),

3. Baake, M., Moody, R.V.: Diffractive point sets with entropy. J. Phys. A: Math. Gen. 31, 9023-9039 (1998), arXiv:math-ph/9809002

4. Baake, M., Höffe, M.: Diffraction of random tilings: some rigorous results. J. Stat. Phys. 99, 219-261 (2000), arXiv:math-ph/9904005

5. Baake, M., Lenz, D.: Dynamical systems on translation bounded measures: pure point dynamical and diffraction spectra. Ergod. Theory Dyn. Syst. 24, 1867-1893 (2004), arXiv:math.DS/0302061

6. Baake, M., Sing, B.: Diffraction spectrum of lattice gas models above $T_{c}$. Lett. Math. Phys. 68, 165-173 (2004), arXiv:math-ph/0405064

7. Baake, M., Lenz, D.: Deformation of Delone dynamical systems and pure point diffraction. J. Fourier Anal. Appl. 11, 125-150 (2005), arXiv:math.DS/0404155

8. Berg, C., Forst, G.: Potential Theory on Locally Compact Abelian Groups. Springer, Berlin (1975)

9. Cowley, J.M.: Diffraction Physics, 3rd edn. North-Holland, Amsterdam (1995)

10. van Enter, A.C.D., Miękisz, J.: How should one define a (weak) crystal? J. Stat. Phys. 66, 1147-1153 (1992)

11. Georgii, H.-O.: Gibbs Measures and Phase Transitions. de Gruyter, Berlin (1988)

12. Guinier, A.: X-Ray Diffraction in Crystals, Imperfect Crystals, and Amorphous Bodies. Dover, New York (1994)

13. Hof, A.: On diffraction by aperiodic structures. Commun. Math. Phys. 169, 25-43 (1995) 
14. Höffe, M., Baake, M.: Surprises in diffuse scattering. Z. Kristallogr. 215, 441-444 (2000), arXiv:math$\mathrm{ph} / 0004022$

15. Kakutani, S.: Strictly ergodic symbolic dynamical systems. In: le Cam, L.M., Neyman, J., Scott, E.L. (eds.) Proceedings of the Sixth Berkeley Symposium on Mathematical Statistics and Probability, pp. 319-326. University of California Press, Berkeley (1972)

16. Keller, G.: Equilibrium States in Ergodic Theory. Cambridge University Press, Cambridge (1998)

17. Külske, C.: Universal bounds on the selfaveraging of random diffraction measures. Probab. Theory Relat. Fields 126, 29-50 (2003), arXiv:math-ph/0109005

18. Külske, C.: Concentration inequalities for functions of Gibbs fields with application to diffraction and random Gibbs measures. Commun. Math. Phys. 239, 29-51 (2003)

19. Lagarias, J.C., Pleasants, P.A.B.: Repetitive Delone sets and quasicrystals. Ergod. Theory Dyn. Syst. 23, 831-867 (2003), arXiv:math.DS/9909033

20. Moody, R.V.: Meyer sets. In: Moody, R.V. (ed.) The Mathematics of Long-Range Aperiodic Order. NATO ASI Series, vol. C489, pp. 403-441. Kluwer, Dordrecht (1997)

21. Moody, R.V.: Model sets: A Survey. In: Axel, F., Dénoyer, F., Gazeau, J.P. (eds.) From Quasicrystals to More Complex Systems, pp. 145-166. EDP Sciences Springer, Les Ulis (2000), arXiv:mathMG/0002020

22. Müller, P., Richard, C.: Random colourings of aperiodic graphs: ergodic and spectral properties. Preprint arXiv:0709.0821 (2007)

23. Rudin, W.: Fourier Analysis on Groups. Wiley, New York (1990)

24. Schlottmann, M.: Generalized model sets and dynamical systems. In: Baake, M., Moody, R.V. (eds.) Directions in Mathematical Quasicrystals. CRM Monograph Series, vol. 13, pp. 143-159. AMS, Providence (2000)

25. Schwartz, L.: Théorie des Distributions, 3nd edn. Hermann, Paris (1998)

26. Welberry, T.R.: Diffuse $X$-Ray Scattering and Models of Disorder. Clarendon, Oxford (2004) 